\title{
A high-quality genome assembly and annotation of the European earwig Forficula auricularia
}

Upendra R. Bhattarai ${ }^{1}$, Mandira Katuwal ${ }^{1}$, Robert Poulin ${ }^{2}$, Neil J. Gemmell ${ }^{1 *}$, Eddy Dowle ${ }^{1 *}$

\author{
${ }^{1}$ Department of Anatomy, University of Otago, Dunedin 9016, New Zealand \\ ${ }^{2}$ Department of Zoology, University of Otago, Dunedin 9016, New Zealand \\ * Corresponding authors
}

Keywords: Forficula auricularia, Hybrid genome assembly, Repeatome, Genome annotation

\begin{abstract}
The European earwig Forficula auricularia is an important model for studies of maternal care, sexual selection, sociality and host-parasite interactions. However, detailed genetic investigations of this species are hindered by a lack of genomic resources. Here we present a high-quality hybrid genome assembly for $F$. auricularia. The genome was assembled using nanopore long-reads and 10x chromium link-reads. The final assembly is $1.06 \mathrm{~Gb}$ in length with $31.03 \%$ GC content. It consists of 919 scaffolds with an N50 of $12.55 \mathrm{Mb}$. Half of the genome is present in only 20 scaffolds. Benchmarking Universal Single-Copy Orthologs scores are $\sim 90 \%$ from three sets of single-copy orthologs (eukaryotic, insect, and arthropod). The total repeat elements in the genome are $64.62 \%$. The MAKER2 pipeline annotated 12,876 proteincoding genes and 21,031 mRNAs. The genome assembly, annotation, and associated resources will be of high value to a large and diverse group of researchers working on Dermapterans.
\end{abstract}

\section{Introduction}

Insects have been at the forefront of genetic research for various biological questions (WilsonSanders 2011; Mukherjee et al. 2015; Simons and Tibbetts 2019). However, most of the genetic studies are carried out on a small number of holometabolous insects that undergo true metamorphosis. In contrast to Holometabola, hemimetabolous insects undergo incomplete metamorphosis with a series of nymphal moults that increasingly resemble the adult form (Truman 2019). It is widely accepted that Holometabola branched out from hemimetabolous ancestors during the Permian 300 Mya (Labandeira and Phillips 1996; Yang 2001). Yet the 
conserved mode of development, embryonic organisation, and the adult body plan of hemimetabolous insects offer a unique model for the study of developmental and evolutionary mechanisms. However, even with the increasing number of sequenced genomes, the majority belong to the Holometabola (Ylla et al. 2021). This has been a bottleneck for the exploration of the diverse biology and life history of hemimetabolous insects. To address this paucity, we report a high-quality annotated genome of the European earwig, Forficula auricularia (Dermaptera: Forficulidae).

The European earwig Forficula auricularia is widely distributed. They are native to the western Eurasian region and were introduced to North America, Australia, and New Zealand, where they have quickly adapted and became abundant throughout the regions (Quarrell et al. 2018; Tourneur and Meunier 2019). Their propensity to dwell on flower and kitchen gardens can cause significant damage to crops, flowers, and commercial vegetables and make them important agricultural pests (Campos et al. 2011; Hill et al. 2019).

They have been of particular interest for many researchers not just because of their importance in the agricultural ecosystem (Binns et al. 2021) but also their importance as a research model for various biological and evolutionary phenomena like sexual selection, maternal care, family interactions, reproductive strategy, and social behaviour (Forslund 2000; Falk et al. 2014; Kramer et al. 2015; Van Meyel and Meunier 2020). They have been extensively studied by behavioural ecologists for the early evolution of group-living and family life (Falk et al. 2014). The male earwigs also show an unusual bias in their use of lateral left and right sexual organs without any conspicuous anatomical differentiation (Kamimura 2006). Like the righthandedness in humans, $90 \%$ of males of giant earwig Labidura riparia show a preference for the right penis for copulation, providing insights into the evolutionary origin of lateralization (Kamimura et al. 2021). Similarly, they are an excellent lab model to study extended phenotypes as they exhibit strange suicidal water-seeking behaviour during the late stages of infection by mermithid nematodes (Herbison et al. 2019). However, their use as a genetic model has been severely limited by the lack of reference genome.

Here, we have sequenced, assembled, annotated, and analysed the genome of the European Earwig, Forficula auricularia. This genome will help researchers study multiple facets of this insect's exciting biology and evolutionary characters and broaden our understanding of insect and genome evolution. 


\section{Methods and Materials}

\section{Sample collection and preparation}

Earwigs (Forficula auricularia) were collected from the Dunedin Botanic Garden $\left(-45^{\circ} 51^{\prime}\right.$ $\left.27.59 " \mathrm{~S}, 170^{\circ} 31 ' 15.56^{\prime \prime} \mathrm{E}\right)$ and reared in a temperature-controlled room (Temperature: cycling from 15 to $12^{\circ} \mathrm{C}$, day/night; Photoperiod of L:D 16:8) in the Department of Zoology, University of Otago, Dunedin. Earwigs were snap-frozen in liquid nitrogen and stored at $-80^{\circ} \mathrm{C}$ before dissection and subsequent nucleotide extraction. Earwigs were dissected in 1x PBS buffer under a dissection microscope to check for nematode parasites, and only non-parasitized individuals were used in this study. The head, wings and muscles from the thorax region were used for DNA extraction. Juvenile instars required for RNA extraction were obtained directly from the field.

\section{DNA extractions}

DNA was extracted using either the Nanobind Tissue Big DNA kit (Circulomics, USA) or DNeasy Blood \& Tissue Kit (Qiagen, Germany) by following the manufacturer's protocol. Tissues from a single individual were used for each extraction. After the extraction, RNase treatment was performed using $4 \mu \mathrm{l}$ of RNase A $(10 \mathrm{mg} / \mathrm{ml})$ per $200 \mu \mathrm{l}$ of DNA elute. DNA was quantified in a Qubit 2.0 Fluorometer (Life Technologies, USA) and quality analysed using Nanodrop. Low quality DNA samples were further cleaned with $1.8 \mathrm{x}$ by volume AMPure XP beads (Beckman Coulter, USA), wherever applicable, following the manufacturer's instructions and eluted in $55 \mu 1$ of molecular grade water. High-quality DNA samples were stored at $-20^{\circ} \mathrm{C}$ and were used within a week of extraction.

\section{Linked read library preparation and sequencing}

Linked read library was prepared at the Genetic Analysis Services (GAS), University of Otago (Dunedin, New Zealand). DNA from an adult male was extracted using the Nanobind kit and size-selected for fragments over 40kbp using Blue Pippin (Sage Science, USA). A chromium 10X linked read (10x Genomics, USA) library was prepared following the manufacturer's instructions. The library was sequenced on the Illumina Nova-seq platform to generate 2x151bp paired-end reads (Garvan Institute, Australia).

\section{Long read library preparation and sequencing}

Five long-read sequencing libraries for Oxford minion were prepared using a ligation sequencing kit (SQK-LSK109) (Oxford Nanopore Technologies, Oxford, UK) following the manufacturer's instructions. To increase the raw nanopore read N50 the first and the second libraries were prepared using 1.75 and $0.75 \mu \mathrm{g}$ of DNA extracted via a Circulomics kit from 
two adult male earwigs. Both libraries were sequenced in a single minion flow cell, flushing the flow cell to remove remains of the first library before loading the second library with a Flow cell wash kit (Oxford Nanopore Technologies, Oxford, UK).

To increase the total raw output the third and the fourth libraries were prepared with DNA from two adult female earwigs, both extracted with a Qiagen kit followed by the AMPure XP beads clean-up step. Input DNA for these two libraries were 2.6 and $3.2 \mu \mathrm{g}$. These were each sequenced on an individual minion flow cell. The fifth library was prepared using $3.0 \mu \mathrm{g}$ of DNA from an adult male earwig. As before DNA was extracted using a Qiagen kit followed by AMPure XP beads clean-up. However, before library preparation, the DNA was sheared five times using a 26G X 0.5 " needle (Terumo, Japan). All prepared libraries were sequenced with R9 chemistry MinION flow cell (FLO-MIN106) (Oxford Nanopore Technologies, UK).

\section{RNA extraction and sequencing}

Total RNA from the different developmental stages, sex, and tissues was extracted using a Direct-zol RNA MicroPrep kit (Zymo Research) with an on filter DNAse treatment following the manufacturer's instructions. Samples included: whole body (gut removed) of juvenile instars 1-2 and juvenile instars 3-4, dissected tissues (antennae, head, thorax, abdomen, legs, and gonads) of adult males and females. RNA from each individual and tissue type was extracted separately. RNA was quantified on a Qubit 2.0 Fluorometer (Life Technologies, USA) and initially quality checked using a nanodrop. Only high-quality extracts were further processed and were stored at $-80^{\circ} \mathrm{C}$ until use.

RNA integrity was evaluated on a Fragment Analyzer (Advanced Analytical Technologies Inc., USA) at the Otago Genomics Facility (OGF), University of Otago, Dunedin, New Zealand. As with most of the insect RNA extracts (Winnebeck et al. 2010) RNA quality number (RQN) values ranged from 2.5 to 10 due to the collapsing of the $28 \mathrm{~S}$ peak; quality was thus determined via the trace rather than RQN. Four pools of samples at equimolar concentration underwent library preparation. Pools consisted of: 8 whole body extractions for juvenile instar 1-2, 8 whole body extractions for juvenile instar 3-4, individual body tissues from five adult males, and individual body tissues from five adult females. TruSeq stranded mRNA libraries were prepared and sequenced as 2x100bp paired-end reads across two lanes of HiSeq 2500 Rapid V2 flowcell at the OGF.

\section{Genome size estimation}

Flow cytometry and k-mer based approach with short-read data were used to estimate the genome size. Flow cytometry analysis was performed on a single head of earwig with two 
biological replicates at Flowjoanna (Palmerston North, NZ). Briefly, the earwig's head was dissociated with a pestle in $500 \mu \mathrm{l}$ of the stock solution containing $0.1 \% \mathrm{w} / \mathrm{v}$ trisodium citrate dihydrate, $0.1 \% \mathrm{v} / \mathrm{v}$ IGEPAL, $0.052 \% \mathrm{w} / \mathrm{v}$ spermine tetrahydrochloride, and $0.006 \%$ sigma 7 9 (all Sigma-Aldrich, USA). Rooster red blood cells (RRBC) from the domestic rooster stored in citrate buffer was used as a reference sample. Test samples were filtered through $35 \mu 1$ filter cap and further dissociated by adding $100 \mu \mathrm{l}$ of $0.21 \mathrm{mg} / \mathrm{ml}$ trypsin followed by $75 \mu 1$ of $2.5 \mathrm{mg} / \mathrm{ml}$ trypsin inhibitor (both Sigma-Aldrich) for 10 minutes at $37^{\circ} \mathrm{C}$. Nuclei were stained using $100 \mu \mathrm{l}$ of pre-stain (containing $416 \mathrm{mg} / \mathrm{ml}$ propidium iodide (PI) with $500 \mathrm{mg} / \mathrm{ml} \mathrm{RNAse}$ in-stock solution). Two sample tubes, one together with prepared RRBC and one without, were then measured on a FACSCalibur (BD Biosciences, USA) equipped with a $488 \mathrm{~nm}$ laser to produce fluorescence collected using the FL-2-Area signal (585/42 BP), along with forward scatter (FSC) and side scatter (SSC) signals which were included for resolving RRBC nuclei from the earwig's nuclei. Data were analysed using Flowjo (BD Biosciences, USA) and the $\mathrm{pg} /$ nuclei of the sample was calculated.

For k-mer based genome size estimation, we used the paired-end linked read sequences. Reads were processed with the scaff_reads script from Scaff10x (v.5.0) to remove the 10x link adapters. Quality control was carried out with Trimmomatic (v.0.39) (options: SLIDINGWINDOW:4:15 LEADING:5 TRAILING:5 MINLEN:35). We used KMC (v.3.1.1) (Kokot et al. 2017) with a k-mer size of 21 to produce a histogram, which was then visualised in Genomescope (v.2.0) web browser.

\section{Bioinformatic pipeline}

All the scripts used for genome assembly, denovo repeat library construction, and annotation are available on GitHub (https://github.com/upendrabhattarai/Earwig_Genome_Project). The bioinformatics software and packages were run in New Zealand eScience Infrastructure (NeSI). Below is a description of the pipeline (Figure 1).

\section{Genome assembly}

Paired-end Illumina reads from the chromium library were assembled using Supernova (v.2.1.1) (Weisenfeld et al. 2017). Assembly quality was assessed using Quast (Gurevich et al. 2013) and BUSCO (Simão et al. 2015) metrics such as N50 values, contig/scaffold number, contiguity and ortholog completeness. Based on several trial assemblies, we down-sampled the total input to 660 million paired-end reads to produce an assembly with better completeness and contiguity. The assembled fasta sequence was obtained with "pseudohap" style of the supernova "mkoutput" function. 
Nanopore reads were basecalled using guppy (v.5.0.7) and assembled using Flye (v.2.7.1) (Kolmogorov et al. 2019) with default parameters. The assembly statistics (as mentioned above) for the Flye assembly were better than that for the Supernova assembly. Therefore, the supernova assembly was merged into the Flye assembly using Quickmerge (Chakraborty et al. 2016). The resulting assembly was processed with Purgehaplotigs (Roach et al. 2018) to remove redundant and duplicated contigs.

The purged genome underwent further scaffolding and gap-closing steps using Rails (v.1.5.1) and Cobbler (v.0.6.1) (Warren 2016), Lrscaf (v.1.1.11) (Qin et al. 2019) and Lrgapcloser (Xu et al. 2019) with the raw long-read data. The resulting assembly was scaffolded with Ragtag (v.2.1.0) (Alonge et al. 2019) using the Supernova assembly. The raw linked-read data were further used to scaffold the assembly with ArbitR (v.0.2) (Hiltunen et al. 2021), Arks (v.1.0.4) (Coombe et al. 2018) and Links (v.1.8.7) (Warren et al. 2015). mRNA-seq reads sequenced for genome annotation purposes, and total RNA-seq reads sequenced for another project (manuscript under preparation) were also used for scaffolding the assembly with the Rascaf (Song et al. 2016). Duplicated and redundant haplotigs were again removed using Purgehaplotigs, and discarded haplotigs were used for scaffolding the assembly using Ragtag again.

Blobtools2 (Laetsch and Blaxter 2017) was used to remove small (<100bp) and low coverage contigs $(<5 \mathrm{x}$ coverage). Contigs that were filtered out were used for re-scaffolding the assembly with Ragtag. Finally, we used Pilon (v.1.24) to polish the assembly using mRNAseq data.

\section{Repeat content analysis}

To assist with annotation a custom repeat library was generated for the Earwig genome using different de novo repeat and homology-based identifiers, including LTRharvest (Ellinghaus et al. 2008), LTRdigest (Steinbiss et al. 2009), RepeatModeler (Flynn et al. 2020), TransposonPSI (Brian Haas 2010) and SINEBase (Vassetzky and Kramerov 2013). We concatenated the individual libraries, and sequences with more than $80 \%$ similarity were merged to remove redundancy using usearch (v.11.0.667) (Edgar 2010). It was then classified using RepeatClassifier. Sequences with unknown categories in the library were mapped against the UniProtKB/Swiss-Prot database (e-value $<1 \mathrm{e}-01$ ); if sequences were not annotated as repeat sequences they were removed from the library. The final repeat library was used in RepeatMasker (v.4.1.2) (Chen 2004) to generate a report for genome repeat content and provided to the Maker2 pipeline to mask the genome. 


\section{Genome annotation}

Genome annotation was carried out with three iterations of the MAKER2 (v.2.31.9) (Holt and Yandell 2011) pipeline combining evidence-based and ab initio gene models. The first round of Maker used evidence-based models and the other two rounds were run using ab initio gene models. For the first round, we provided the MAKER2 pipeline with 180,119 mRNA transcripts denovo assembled via the Trinity pipeline (Grabherr et al. 2011) along with 26,414 mRNA and 1,529 protein sequences of dermapterans from NCBI and 779 dermapteran protein sequences from the Uniprot database.

Augustus was trained using Braker (v.2.16) (Hoff et al. 2019) and SNAP was trained after each round of MAKER to use for ab initio gene model prediction. For the functional annotation, we ran InterProScan (v.5.51-85.0) (Jones et al. 2014) for the predicted protein sequences obtained from MAKER and retrieved InterPro ID, PFAM domains, and Gene Ontology (GO) terms. Furthermore, we ran BLAST with the Uniprot database to assign gene descriptors to each transcript based on the best BLAST hit.

\section{Results and Discussion}

\section{Genome size estimates}

The flow cytometer estimated the genome size of $968.22 \pm 20.747 \mathrm{Mb}$ (Mean $\pm \mathrm{SD}$ ) for the earwig genome. Similarly, the kmer based approach using adapter removed paired-end data from linked read sequencing estimated the male earwig to be $988 \mathrm{Mb}$. Whereas, an earlier estimation of a unknown dermapteran (earwig) species genome size was 1.4 Gb (Gregory 2005) showing a variable genome size within the order.

\section{Genome assembly}

A total of 799.6 million paired-end reads was generated for the linked-read library. Downsampled to 660 million paired-end reads, Supernova estimated the genome size of 1.22 $\mathrm{Gb}$, raw coverage of $82.02 \%$, effective coverage of $39.50 \%$ and weighted mean molecule size of $22.45 \mathrm{~kb}$. The Supernova assembly was $1.15 \mathrm{~Gb}$ in size and had 145,055 contigs, with an $\mathrm{N} 50$ of $0.03 \mathrm{Mb}$ and L50 of 7,500. Quast reported a complete Busco of $64.69 \%$ and a partial Busco of $9.24 \%$ from the eukaryotic database.

The nanopore sequencing yielded approximately $10.7 \mathrm{~Gb}$ of data, consisting of over 3 million reads. The median read length was 897 bp with an N50 length of 11,986 bp (Supplementary Table 1). The median read Phred quality was 13.34. Primary assembly from Flye produced an assembly of $1.1 \mathrm{~Gb}$. There were 187,66 contigs with $\mathrm{N} 50$ of $0.18 \mathrm{Mb}$ and L50 of 1,832. Quast reported a complete Busco of $82.18 \%$ and a partial Busco of $9.24 \%$. The long-read assembly 
showed better contiguity and completeness, so we merged the linked-read assembly with the long-read assembly (Table 1).

The final hybrid assembly has a size of $1.06 \mathrm{~Gb}$. It has 919 scaffolds with an N50 of $12.55 \mathrm{Mb}$, which shows that the assembly is highly contiguous. Half of the genome is present in just 20 scaffolds, as denoted by the L50 number (Table 1). Assembly has 846.85 "N's" per 100kbp. The Busco score from the insect database $(n=1,367)$ for the assembly was $87.1 \%$ complete, among which $4.1 \%$ were duplicated, and 3.1\% fragmented Busco (Figure 2). Improvement in assembly statistics after each processing step is given in Supplementary Table 2.

The only other whole-genome sequence publicly available from the Dermaptera order is of the other earwig Anisolabis maritima. Using the insect database $(n=1,367)$ it has $83.4 \%$ complete and $10.8 \%$ fragmented Busco scores. The A. maritima assembly has an N50 of $1.4 \mathrm{Mb}$ with a genome size of $649.7 \mathrm{Mb}$ (Mei et al. 2022). So, in comparison, the F. auricularia genome assembly has a better gene model and contiguity from the Dermaptera order.

\section{Genome repeat contents}

Repeat analysis of the assembly showed that interspersed repeats comprised $\sim 686 \mathrm{Mb}$ $(64.62 \%)$ of the F. auricularia genome. This includes $\sim 248 \mathrm{Mb}$ of retroelements $(23.37 \%$ of the genome), $\sim 178 \mathrm{Mb}$ of DNA transposons (16.79\% of the genome), $\sim 35 \mathrm{Mb}$ of rolling-circles (3.28\% of the genome) and $260 \mathrm{Mb}$ of unclassified elements (Table 2). Unusually large and variable genome sizes characterise Hemimetabolans (Wu et al. 2017). Comparative analysis in six species of Gomphocerine grasshoppers showed a strong positive correlation between repeat content and genome size. Genome size ranged from 8.2 to $13.7 \mathrm{~Gb}$ in these six species with a repeat content ranging routinely from $79 \%$ to $87 \%$, with the exception of Stauroderus scalaris whose genome is $96 \%$ repetitive DNA and the second-largest insect genome documented. Our estimation of genome size for $F$. auricularia doesn't show gigantism $(\sim 968.22 \mathrm{Mb}$, flow cytometer estimate). However, its repeatome (64.62\%), is almost twice that of other hemimetabolous insects like Gryllus bimaculatus (33.69\%) and Laupala kohalensis (35.51\%) (Ylla et al. 2021). This fold increase in the repeatome is surprising given both G. bimaculatus and L. kohalensis have bigger genomes $(\sim 1.6 \mathrm{~Gb})$ than $F$. auricularia.

\section{Genome annotation}

Combining evidence-based and $a b$ initio gene models in the MAKER2 pipeline, we identified 12,876 genes and $21,031 \mathrm{mRNAs}$ in the genome assembly. The mean gene length is $12,096 \mathrm{bp}$ and the total gene length is $155.75 \mathrm{Mb}$, which makes $14.7 \%$ of the whole assembly. The longest gene annotated is $412,198 \mathrm{bp}$ and the longest CDS is 19,035 bp (Table 3). 61.35\% of total predicted mRNAs and $59.53 \%$ of predicted proteins are also functionally annotated through 
either one or more of InterPro, Gene ontology and Pfam databases (Supplementary Table 3). Comparing with the Arthropoda database we got $74.4 \%$ complete Busco score for annotated Transcriptome and 70.9\% complete Busco score for annotated Proteins (Figure 3). 98.3\% of the gene models have AED score of 0.5 or less, assuring highly confident gene prediction (Supplementary Figure 1).

The GC content of the $F$. auricularia genome is $31.03 \%$, markedly greater than that the $19.3 \%$ GC observed in the genome of the earwig A. maritima (Mei et al. 2022). A comparison of GC content for different genomic regions of $F$. auricularia showed that exons have higher GC content $(0.372 \pm 0.087)($ Mean \pm SD) and introns have lower $(0.267 \pm 0.075)$ when compared between intergenic regions $(\mathrm{N}=1,565)$, genes $(\mathrm{N}=12,876)$, exons $(\mathrm{N}=145,003)$, introns $(\mathrm{N}$ $=123,973)$, and non-overlapping $10 \mathrm{~kb}$ windows throughout the genome $(\mathrm{N}=106,686)$ (Figure 4). GC content for $10 \mathrm{~kb}$ windows was $0.308 \pm 0.032$ which resembles the mean GC content of the whole genome (0.310). This finding is not unexpected as a higher GC content in exons versus introns is common across the animal and plant kingdom because of the evolutionary selection of exon regions (Amit et al., 2012). There was significant different for each pairwise comparison using Anova followed by Tukey HSD with $P<0.01$ for Intergenic vs $10 \mathrm{~kb}$ window and $P<0.0001$ for all others.

In conclusion, the assembled and annotated $F$. auricularia genome presented here is a key resource to develop this important insect species as a genetic model. We anticipate this will enhance the genetic study on various aspects of its biology, including developmental biology, sociality, and evolutionary characteristics.

\section{Data Availability Statement}

The genome assembly and annotation of Forficula auricularia are available through FigShare https://doi.org/10.6084/m9.figshare.19092044. The raw sequencing reads are deposited in NCBI with accession number PRJNA800435.

\section{Code availability}

The scripts used for genome assembly, repeat library preparation and masking, and genome annotation, are available at GitHub (https://github.com/upendrabhattarai/Earwig_genome_project) under GNU GPLv3 license. 


\section{Acknowledgements}

We thank the support team at New Zealand eScience Infrastructure (NeSI) for their help running various software on their HPC platforms and the staff at Dunedin Botanical Garden, Dunedin, New Zealand, for their help during earwig collection in the field.

\section{Conflict of Interest}

The authors declare no competing interests.

\section{Funder Information}

This study was funded by the Royal Society Te Apārangi Marsden Fund grant (16-UOO-152).

\section{Literature Cited}

Alonge, M., S. Soyk, S. Ramakrishnan, X. Wang, S. Goodwin et al., 2019 RaGOO: fast and accurate reference-guided scaffolding of draft genomes. Genome Biol. 20: 224.

Binns, M., A. A. Hoffmann, M. Helden, T. Heddle, M. P. Hill et al., 2021 Lifecycle of the invasive omnivore, Forficula auricularia, in Australian grain growing environments. Pest Manag. Sci. 77: 1818-1828.

Brian Haas, 2010 TransposonPSI: An Application of PSI-Blast to Mine (Retro-)Transposon ORF Homologies.

Campos, M. R., M. C. Picanço, J. C. Martins, A. C. Tomaz, and R. N. C. Guedes, 2011 Insecticide selectivity and behavioral response of the earwig Doru luteipes. Crop Prot. 30: $1535-1540$.

Chakraborty, M., J. G. Baldwin-Brown, A. D. Long, and J. J. Emerson, 2016 Contiguous and accurate de novo assembly of metazoan genomes with modest long read coverage. Nucleic Acids Res. 44: gkw654.

Chen, N., 2004 Using RepeatMasker to Identify Repetitive Elements in Genomic Sequences. Curr. Protoc. Bioinforma. 5: 4.10.1-4.10.14.

Coombe, L., J. Zhang, B. P. Vandervalk, J. Chu, S. D. Jackman et al., 2018 ARKS: chromosome-scale scaffolding of human genome drafts with linked read kmers. BMC Bioinformatics 19: 234.

Edgar, R. C., 2010 Search and clustering orders of magnitude faster than BLAST. Bioinformatics 26: 2460-2461.

Ellinghaus, D., S. Kurtz, and U. Willhoeft, 2008 LTRharvest, an efficient and flexible software for de novo detection of LTR retrotransposons. BMC Bioinformatics 9: 18. 
Falk, J., J. W. Y. Wong, M. Kölliker, and J. Meunier, 2014 Sibling Cooperation in Earwig Families Provides Insights into the Early Evolution of Social Life. Am. Nat. 183: 547557.

Flynn, J. M., R. Hubley, C. Goubert, J. Rosen, A. G. Clark et al., 2020 RepeatModeler2 for automated genomic discovery of transposable element families. Proc. Natl. Acad. Sci. 117: 9451-9457.

Forslund, P., 2000 Male-male competition and large size mating advantage in European earwigs, Forficula auricularia. Anim. Behav. 59: 753-762.

Grabherr, M. G., B. J. Haas, M. Yassour, J. Z. Levin, D. A. Thompson et al., 2011 Fulllength transcriptome assembly from RNA-Seq data without a reference genome. Nat. Biotechnol. 29: 644-652.

Gregory, T. R., 2005 Genome Size Evolution in Animals, pp. 3-87 in The Evolution of the Genome, edited by T. R. B. T.-T. E. of the G. Gregory. Elsevier, Burlington.

Gurevich, A., V. Saveliev, N. Vyahhi, and G. Tesler, 2013 QUAST: quality assessment tool for genome assemblies. Bioinformatics 29: 1072-1075.

Herbison, R. E. H., S. Evans, J.-F. Doherty, and R. Poulin, 2019 Let's go swimming: mermithid-infected earwigs exhibit positive hydrotaxis. Parasitology 146: 1631-1635.

Hill, M. P., M. Binns, P. A. Umina, A. A. Hoffmann, and S. Macfadyen, 2019 Climate, human influence and the distribution limits of the invasive European earwig, Forficula auricularia, in Australia. Pest Manag. Sci. 75: 134-143.

Hiltunen, M., M. Ryberg, and H. Johannesson, 2021 ARBitR: an overlap-aware genome assembly scaffolder for linked reads (M. Pier Luigi, Ed.). Bioinformatics 37: 22032205.

Hoff, K. J., A. Lomsadze, M. Borodovsky, and M. Stanke, 2019 Whole-Genome Annotation with BRAKER, pp. 65-95 in Methods in molecular biology (Clifton, N.J.),.

Holt, C., and M. Yandell, 2011 MAKER2: an annotation pipeline and genome-database management tool for second-generation genome projects. BMC Bioinformatics 12: 491.

Jones, P., D. Binns, H.-Y. Chang, M. Fraser, W. Li et al., 2014 InterProScan 5: genome-scale protein function classification. Bioinformatics 30: 1236-1240.

Kamimura, Y., 2006 Right-handed penises of the earwig Labidura riparia (Insecta, Dermaptera, Labiduridae): Evolutionary relationships between structural and behavioral asymmetries. J. Morphol. 267: 1381-1389.

Kamimura, Y., Y. Matsumura, C.-C. S. Yang, and S. N. Gorb, 2021 Random or handedness? Use of laterally paired penises in Nala earwigs (Insecta: Dermaptera: Labiduridae). Biol. J. Linn. Soc. 134: 716-731.

Kokot, M., M. Długosz, and S. Deorowicz, 2017 KMC 3: counting and manipulating k-mer 
statistics (B. Berger, Ed.). Bioinformatics 33: 2759-2761.

Kolmogorov, M., J. Yuan, Y. Lin, and P. A. Pevzner, 2019 Assembly of long, error-prone reads using repeat graphs. Nat. Biotechnol. 37: 540-546.

Kramer, J., J. Thesing, and J. Meunier, 2015 Negative association between parental care and sibling cooperation in earwigs: a new perspective on the early evolution of family life? J. Evol. Biol. 28: 1299-1308.

Labandeira, C. C., and T. L. Phillips, 1996 A Carboniferous insect gall: insight into early ecologic history of the Holometabola. Proc. Natl. Acad. Sci. 93: 8470-8474.

Laetsch, D. R., and M. L. Blaxter, 2017 BlobTools: Interrogation of genome assemblies. F1000Research 6: 1287.

Mei, Y., D. Jing, S. Tang, X. Chen, H. Chen et al., 2022 InsectBase 2.0: a comprehensive gene resource for insects. Nucleic Acids Res. 50: D1040-D1045.

Van Meyel, S., and J. Meunier, 2020 Filial egg cannibalism in the European earwig: its determinants and implications in the evolution of maternal egg care. Anim. Behav. 164: $155-162$.

Mukherjee, K., R. M. Twyman, and A. Vilcinskas, 2015 Insects as models to study the epigenetic basis of disease. Prog. Biophys. Mol. Biol. 118: 69-78.

Qin, M., S. Wu, A. Li, F. Zhao, H. Feng et al., 2019 LRScaf: improving draft genomes using long noisy reads. BMC Genomics 20: 955.

Quarrell, S. R., J. Arabi, A. Suwalski, M. Veuille, T. Wirth et al., 2018 The invasion biology of the invasive earwig, Forficula auricularia in Australasian ecosystems. Biol. Invasions 20: $1553-1565$.

Roach, M. J., S. A. Schmidt, and A. R. Borneman, 2018 Purge Haplotigs: allelic contig reassignment for third-gen diploid genome assemblies. BMC Bioinformatics 19: 460.

Simão, F. A., R. M. Waterhouse, P. Ioannidis, E. V. Kriventseva, and E. M. Zdobnov, 2015 BUSCO: assessing genome assembly and annotation completeness with single-copy orthologs. Bioinformatics 31: 3210-3212.

Simons, M., and E. Tibbetts, 2019 Insects as models for studying the evolution of animal cognition. Curr. Opin. Insect Sci. 34: 117-122.

Song, L., D. S. Shankar, and L. Florea, 2016 Rascaf: Improving Genome Assembly with RNA Sequencing Data. Plant Genome 9:.

Steinbiss, S., U. Willhoeft, G. Gremme, and S. Kurtz, 2009 Fine-grained annotation and classification of de novo predicted LTR retrotransposons. Nucleic Acids Res. 37: 70027013.

Tourneur, J.-C., and J. Meunier, 2019 Thermal regimes, but not mean temperatures, drive 
patterns of rapid climate adaptation at a continent-scale: evidence from the introduced European earwig across North America. bioRxiv 550319.

Truman, J. W., 2019 The Evolution of Insect Metamorphosis. Curr. Biol. 29: R1252-R1268.

Vassetzky, N. S., and D. A. Kramerov, 2013 SINEBase: a database and tool for SINE analysis. Nucleic Acids Res. 41: D83-D89.

Warren, R. L., 2016 RAILS and Cobbler: Scaffolding and automated finishing of draft genomes using long DNA sequences. J. Open Source Softw. 1: 116.

Warren, R. L., C. Yang, B. P. Vandervalk, B. Behsaz, A. Lagman et al., 2015 LINKS: Scalable, alignment-free scaffolding of draft genomes with long reads. Gigascience 4: 35 .

Weisenfeld, N. I., V. Kumar, P. Shah, D. M. Church, and D. B. Jaffe, 2017 Direct determination of diploid genome sequences. Genome Res. 27: 757-767.

Wilson-Sanders, S. E., 2011 Invertebrate Models for Biomedical Research, Testing, and Education. ILAR J. 52: 126-152.

Winnebeck, E. C., C. D. Millar, and G. R. Warman, 2010 Why Does Insect RNA Look Degraded? J. Insect Sci. 10: 1-7.

Wu, C., V. G. Twort, R. N. Crowhurst, R. D. Newcomb, and T. R. Buckley, 2017 Assembling large genomes: analysis of the stick insect (Clitarchus hookeri) genome reveals a high repeat content and sex-biased genes associated with reproduction. BMC Genomics 18: 884 .

Xu, G.-C., T.-J. Xu, R. Zhu, Y. Zhang, S.-Q. Li et al., 2019 LR_Gapcloser: a tiling pathbased gap closer that uses long reads to complete genome assembly. Gigascience 8:

Yang, A. S., 2001 Modularity, evolvability, and adaptive radiations: a comparison of the hemi- and holometabolous insects. Evol. Dev. 3: 59-72.

Ylla, G., T. Nakamura, T. Itoh, R. Kajitani, A. Toyoda et al., 2021 Insights into the genomic evolution of insects from cricket genomes. Commun. Biol. 4: 733. 


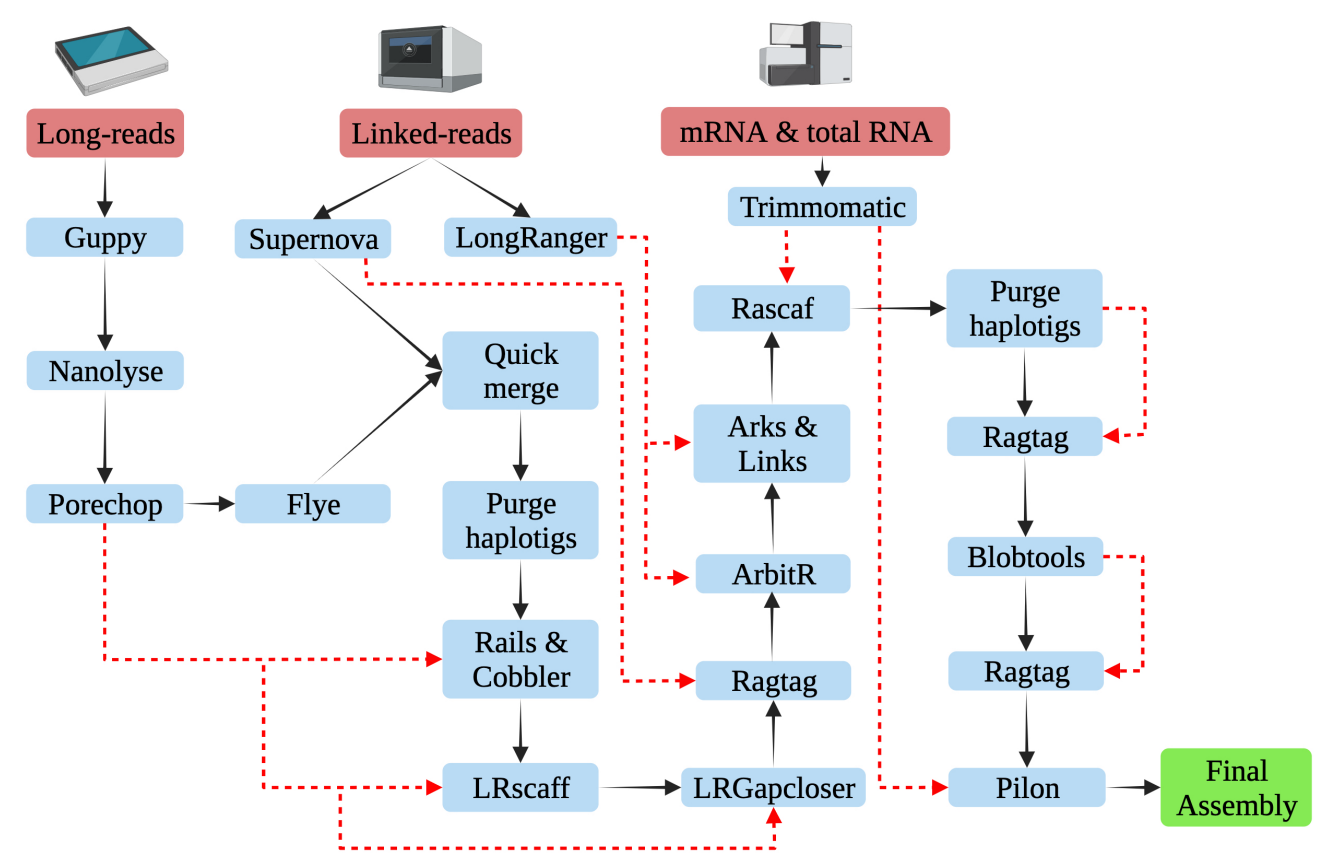

Figure 1: Schematic representation of the assembly pipeline for the Forficula auricularia genome. The black arrow represents the workflow and the red dotted lines represent the additional input data in the pipeline. 


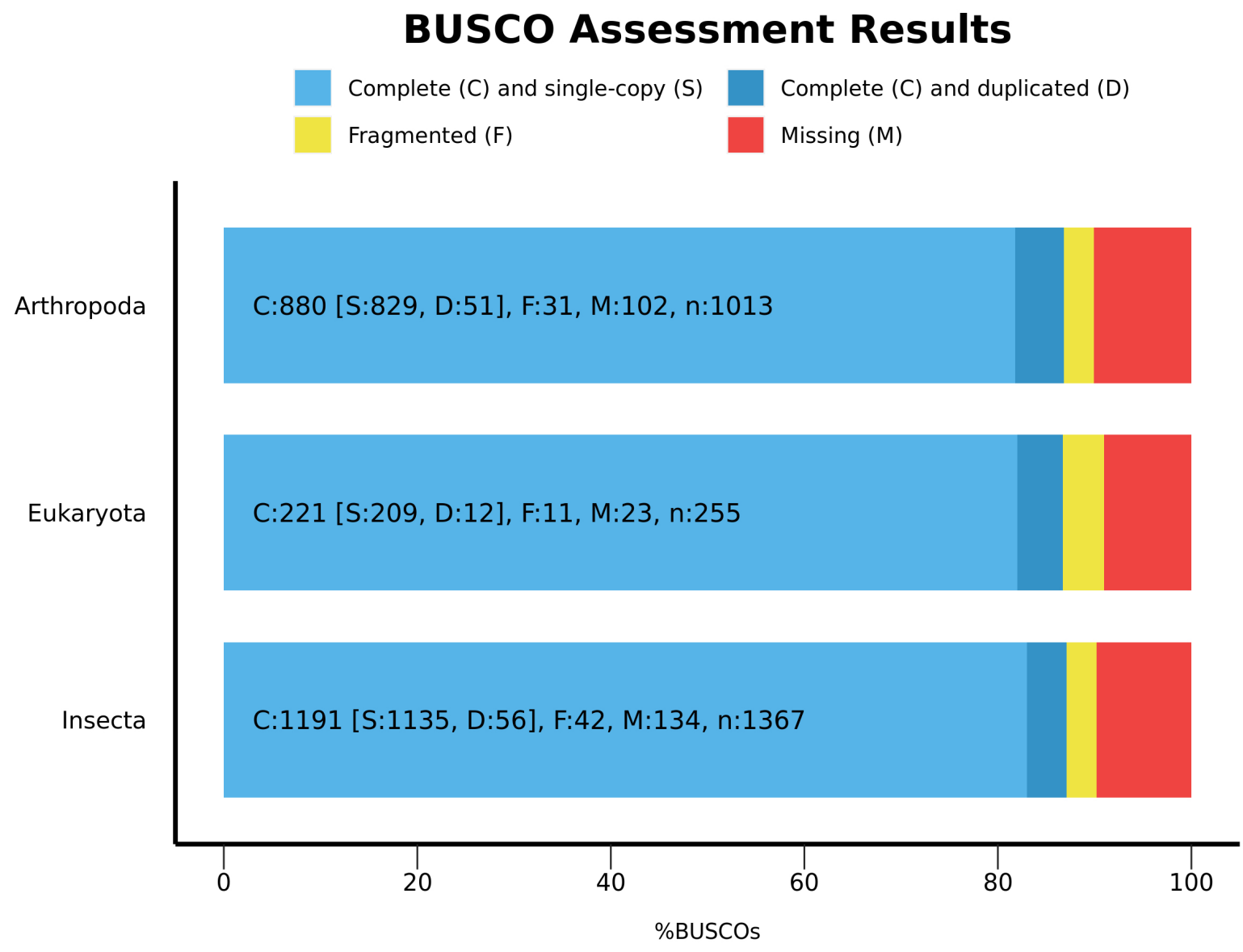

Figure 2. The Busco (v.5) report for the final hybrid assembly of the Forficula auricularia genome. Busco scores in percentage (x-axis) from Arthropoda, Eukaryota, and Insecta (odt10) databases (y-axis) are shown in the bar plot. The light blue portion of the bar represents Complete and single-copy orthologs, dark blue represents complete and duplicated orthologs, yellow represents fragmented Busco genes and red represents missing Busco genes. 


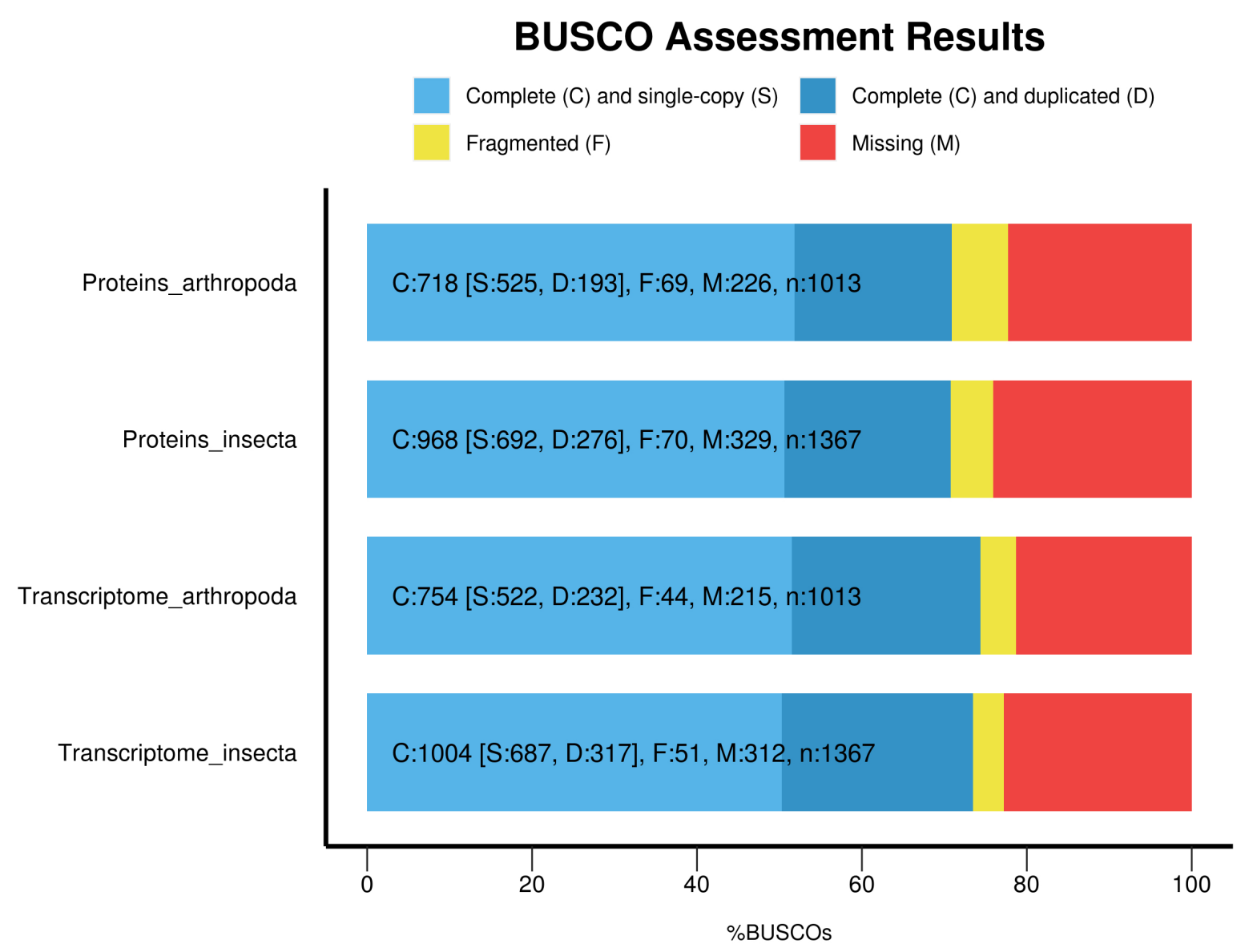

Figure 3. Annotation completeness through Busco database. The plot shows the Busco (v.5) percentage (x-axis) for the annotated Proteins and Transcriptomes using Arthropoda_odb10 and Insecta_odb10 database as indicated in the y-axis. 


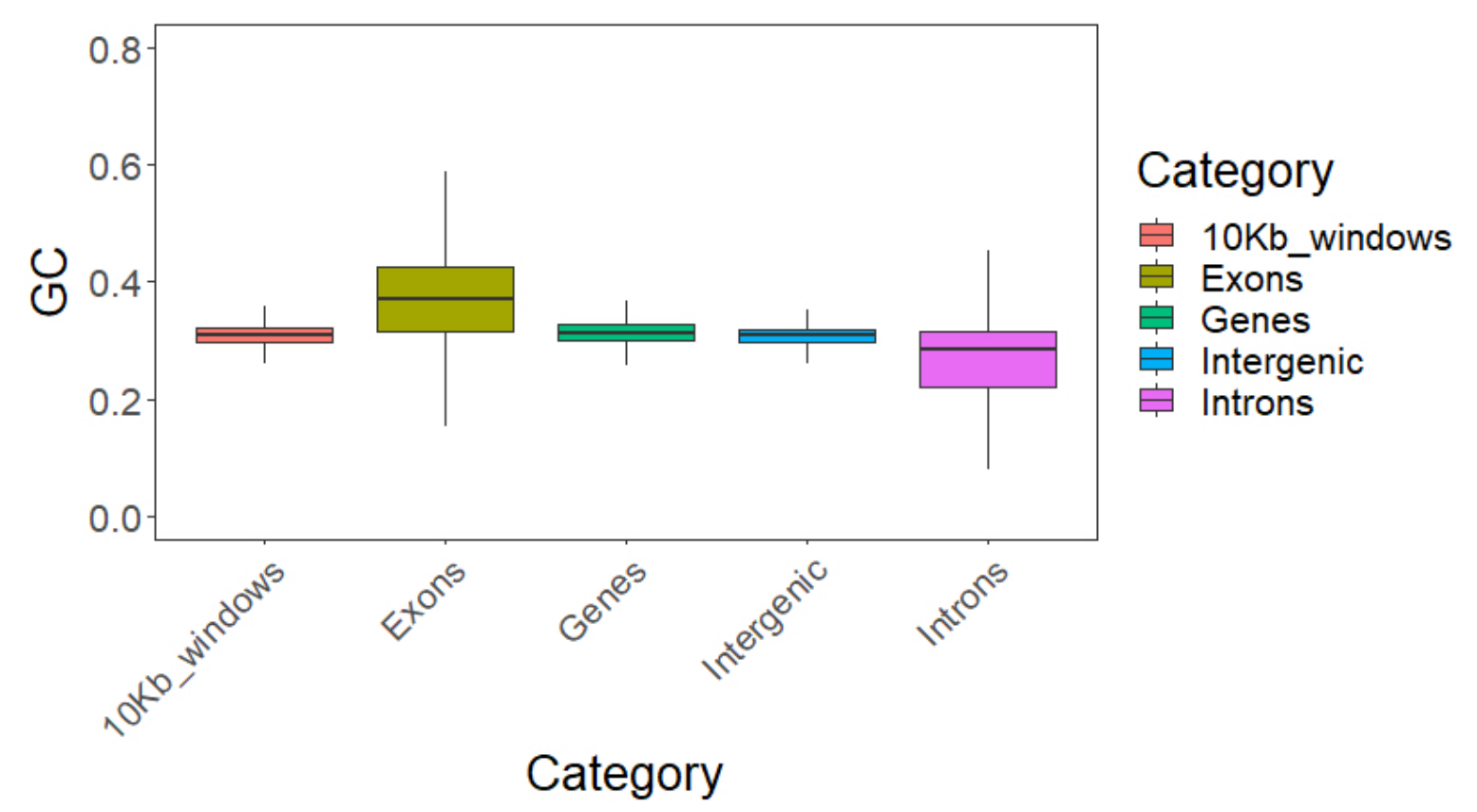

Figure 4. GC percentage in different genomic regions in F. auricularia genome. GC content for $10 \mathrm{~kb}$ windows were generated without regards to any genomic features. Whiskers extend to 25 th and 75 th percentiles. GC content in exons is higher and in introns is lower compared to genome average. 
Table 1. Assembly statistics for the genome assembly of the European earwig Forficula auricularia. Quast scores are to its default Eukaryota database.

\begin{tabular}{|l|c|c|c|c|c|c|c|}
\hline & $\begin{array}{c}\text { Assembly } \\
\text { length }\end{array}$ & $\begin{array}{c}\text { No. of } \\
\text { scaffolds }\end{array}$ & N50 & L50 & $\begin{array}{c}\text { Ns per } \\
\text { 100 kbp }\end{array}$ & \multicolumn{2}{|c|}{ Busco \% (Quast) } \\
\cline { 5 - 8 } & & & & Complete & Partial \\
\hline $\begin{array}{l}\text { Supernova } \\
\text { assembly }\end{array}$ & $1,145,470,221$ & 145,055 & 30,358 & 7,500 & $3,677.89$ & 64.69 & 9.24 \\
\hline $\begin{array}{l}\text { Flye } \\
\text { assembly }\end{array}$ & $1,118,374,848$ & 18,766 & 180,737 & 1,832 & 0.35 & 82.18 & 9.24 \\
\hline $\begin{array}{l}\text { Final } \\
\text { hybrid } \\
\text { assembly }\end{array}$ & $1,062,210,345$ & 919 & $12,548,649$ & 20 & 846.85 & 87.13 & 2.97 \\
\hline
\end{tabular}

Table 2. Repeat content analysis in the European earwig Forficula auricularia genome.

\begin{tabular}{|c|c|c|c|c|}
\hline No. of sequences: & & & & 919 \\
\hline Total length (bp): & \multicolumn{4}{|r|}{1062210345} \\
\hline GC level: & \multicolumn{4}{|r|}{$31.03 \%$} \\
\hline Bases masked: & \multicolumn{4}{|c|}{$722769501 \mathrm{bp}(68.04 \%)$} \\
\hline & & Numbers & Length (bp) & Percentage \\
\hline \multirow[t]{14}{*}{ Retroelements } & & 1385007 & 248236495 & $23.37 \%$ \\
\hline & SINEs: & 41157 & 5138497 & $0.48 \%$ \\
\hline & Penelope & 50409 & 10372837 & $0.98 \%$ \\
\hline & LINEs: & 660178 & 124985146 & $11.77 \%$ \\
\hline & CRE/SLACS & 0 & 0 & $0.00 \%$ \\
\hline & L2/CR1/Rex & 112418 & 20654321 & $1.94 \%$ \\
\hline & R1/LOA/Jockey & 167317 & 22277052 & $2.10 \%$ \\
\hline & $\mathrm{R} 2 / \mathrm{R} 4 / \mathrm{NeSL}$ & 23348 & 4271189 & $0.40 \%$ \\
\hline & RTE/Bov-B & 136406 & 28799096 & $2.71 \%$ \\
\hline & L1/CIN4 & 10079 & 1892539 & $0.18 \%$ \\
\hline & LTR elements: & 683672 & 118112852 & $11.12 \%$ \\
\hline & $\mathrm{BEL} / \mathrm{Pao}$ & 60561 & 12114300 & $1.14 \%$ \\
\hline & Ty1/Copia & 97132 & 14352992 & $1.35 \%$ \\
\hline & Gypsy/DIRS1 & 521467 & 91083363 & $8.57 \%$ \\
\hline
\end{tabular}




\begin{tabular}{|c|c|c|c|c|}
\hline & Retroviral & 3701 & 443583 & $0.04 \%$ \\
\hline DNA transposons & & 1040870 & 178326460 & $16.79 \%$ \\
\hline & hobo-Activator & 362395 & 59188939 & $5.57 \%$ \\
\hline & Tc1-IS630-Pogo & 355781 & 66331225 & $6.24 \%$ \\
\hline & En-Spm & 0 & 0 & $0.00 \%$ \\
\hline & MuDR-IS905 & 0 & 0 & $0.00 \%$ \\
\hline & PiggyBac & 21153 & 2726812 & $0.26 \%$ \\
\hline & Tourist/Harbinger & 5541 & 1187174 & $0.11 \%$ \\
\hline & Other (Mirage, & 10240 & 1580945 & $0.15 \%$ \\
\hline & $\begin{array}{l}\text { P-element, } \\
\text { Transib) }\end{array}$ & & & \\
\hline Rolling-circles & & 174964 & 34830487 & $3.28 \%$ \\
\hline Unclassified: & & 1563937 & 259874747 & $24.47 \%$ \\
\hline $\begin{array}{l}\text { Total interspersed } \\
\text { repeats: }\end{array}$ & & & 686437702 & $64.62 \%$ \\
\hline Small RNA: & & 9913 & 1406877 & $0.13 \%$ \\
\hline Satellites: & & 1110 & 495561 & $0.05 \%$ \\
\hline Simple repeats: & & $\mathbf{0}$ & $\mathbf{0}$ & $0.00 \%$ \\
\hline Low complexity: & & $\mathbf{0}$ & $\mathbf{0}$ & $0.00 \%$ \\
\hline
\end{tabular}

Table 3. Genome annotation summary for the European earwig Forficula auricularia

\begin{tabular}{|l|l|}
\hline Total sequence length & $1,062,210,345$ \\
\hline Number of genes & 12,876 \\
\hline Number of mRNAs & 21,031 \\
\hline Number of exons & 145,003 \\
\hline Number of introns & 123,973 \\
\hline Number of CDS & 21,030 \\
\hline Total gene length & $155,753,058$ \\
\hline
\end{tabular}




\begin{tabular}{|c|c|}
\hline Total mRNA length & $271,884,000$ \\
\hline Total exon length & $32,584,454$ \\
\hline Total intron length & $239,538,939$ \\
\hline Total CDS length & $23,936,568$ \\
\hline Shortest gene & 3 \\
\hline Shortest mRNA & 3 \\
\hline Shortest exon & 1 \\
\hline Shortest intron & 4 \\
\hline Shortest CDS & 3 \\
\hline Longest gene & 412,198 \\
\hline Longest mRNA & 412,198 \\
\hline Longest exon & 10,240 \\
\hline Longest intron & 319,382 \\
\hline Longest CDS & 19,035 \\
\hline mean gene length & 12,096 \\
\hline mean mRNA length & 12,928 \\
\hline mean exon length & 225 \\
\hline mean intron length & 1,932 \\
\hline mean CDS length & 1,138 \\
\hline$\%$ of genome covered by genes & 14.7 \\
\hline$\%$ of genome covered by CDS & 2.3 \\
\hline mean mRNAs per gene & 2 \\
\hline mean exons per mRNA & 7 \\
\hline mean introns per mRNA & 6 \\
\hline
\end{tabular}

